\title{
Optimum Watermark Detection in Color Images
}

\author{
Elisa Sayrol, Josep Vidal, Silvia Cabanillas, Sonia Santamaria \\ Dept. Teoría de la Señal y Comunicaciones \\ Universidad Politécnica de Cataluña \\ Campus Nord, Módulo D5, c/ Jordi Girona 1-3 \\ 08034 Barcelona, SPAIN \\ Tel : 34-93-4016457. Fax : 34-93-4016447 \\ Email: \{elisa,pepe\}@gps.tsc.upc.es
}

\begin{abstract}
This work concentrates on the problem of watermarking embedding and optimum detection in. color images through the use of spread spectrum techniques, both in space (Direct Sequence Spread Spectrum or DSSS) and frequency (Frequency Hopping). It is applied to $R G B$ and opponent color component representations. Perceptive information is considered in both color systems. Some tests are performed in order to ensure imperceptibility and to assess detection quality of the optimum color detectors
\end{abstract}

\section{Introduction}

The watermark system described in [2] was successfully applied to grey scale images and could be easily extended to color images by embedding the watermark in the luminance component. Actually this strategy for color images is often used in the literature. Some efforts have been done to derive watermark systems specifically considering color images. Thus, in [3] the watermark is added in the blue channel motivated by the fact that the human visual system is less sensitive to this component. In [4] a model of human color vision based on the CIELAB standard is used and the hidden information is embedded in the yellow-blue opponent component. Nevertheless, there is no need to limit the system to watermark only one component, the perceptual model will correctly weight the amplitude of the embedded signal in each component in order to preserve imperceptibility. The objectives may be the introduction of more information in the mark or the decrease in the probability of error by the diversity of including the mark in three components.
In [1] optimum detectors were derived in the ML sense for the case of grey-scaled images. However the extension to color images is not straightforward when the watermark is added to each component. In this work we derive optimum detectors for color images. Perceptual models are also used to ensure the invisibility of the watermark, in particular the perceptual models described in [3] and [4] are considered.

\section{Digital Watermarks}

There are many techniques that successfully embed hidden information in an image. In particular, spread spectrum techniques in space (DSSS) and in frequency (FH) adapt specifically to the different requirements of a watermarking system [5]. In this work we concentrate on two important aspects that affect color images.

First, the probability of error in the detection of each symbol constituting the watermark should be as low as possible, implying high power for the mark and, at the same time, noticeable effect on the marked image. The use of optimum detectors exclusively designed for color images allows maintaining the probability of error while reducing the amplitude of the embedded signal.

Second, visual quality of the marked image should be indistinguishable from the original. The contribution in [2] is a pioneering work on the use of luminance psychovisual criteria in the watermark embedding process, by modeling the behavior of human visual system with Gabor filters. On the other hand the S-CIELAB system is an extension of CIELAB which is based on psychophysical studies of color discriminability and takes spatial structure also into account [3]. This system can be used to measure and reduce perceptibility in watermarked color images. 


\section{Optimum Watermark Detection in Color Images}

It is required that the authorized user be able to recover the watermark with low probability of error. Good detection schemes allow the watermark embedding with low power and hence, low visual impact. Before deriving the detector, let us formulate a model for the watermark: if we do represent the watermarked color image in vector notation, as :

$\boldsymbol{r}=\boldsymbol{p}+\mu \circ \boldsymbol{q}_{i}$ where

$\boldsymbol{r}=\left[\begin{array}{l}\mathbf{r}_{R} \\ \mathbf{r}_{G} \\ \mathbf{r}_{B}\end{array}\right], \boldsymbol{p}=\left[\begin{array}{l}\mathbf{p}_{\mathbf{R}} \\ \mathbf{p}_{\mathbf{G}} \\ \mathbf{p}_{\mathbf{B}}\end{array}\right], \mu \circ \boldsymbol{q}_{i}=\left[\begin{array}{c}\mu_{R} \mathbf{q}_{R i} \\ \mu_{G} \mathbf{q}_{G i} \\ \mu_{B} \mathbf{q}_{B i}\end{array}\right]$,

and $\circ$ stands for element-to element vector product.

$\boldsymbol{r}$ : Watermarked image with three components. In the following derivations, RGB components are used although the same derivations apply when other color components are used, for example opponent components. $p$ : Original three components image.

$\mu$ : Amplitude that controls the total power for each component.

$\boldsymbol{q}_{\mathrm{i}}$ : Weighted symbol, that is, for each component $\boldsymbol{X}$ we have: $\boldsymbol{q}_{X i}=\mathbf{f}_{\mathbf{x}} \circ \mathbf{s}_{i}$ where $\mathbf{f}_{\mathbf{x}}$ corresponds to the perceptual mask and $\mathbf{s}_{i}$ stands for the original symbol to code, which is a pseudorandom minimum length sequence [1].

\subsection{Optimum linear detector}

The optimum detector has to take into account the density function of the noise, that is, the image $p$. The conventional and computationally simple approach is to consider the noise to be Gaussian and stationary. In this case, the log-likelihood function of $\boldsymbol{r}$ is given by:

$$
\Psi=\left(\boldsymbol{r}-\boldsymbol{H}_{\boldsymbol{i}} \boldsymbol{\theta}\right)^{T} \boldsymbol{C}_{\boldsymbol{R} G \boldsymbol{B}}^{-1}\left(\boldsymbol{r}-\boldsymbol{H}_{\boldsymbol{i}} \boldsymbol{\theta}\right)
$$

The detectors derived in the following make the approximation that the $f_{x}$ amplitudes are constant (not space dependant). This translates into 3-components for the vector $\theta$.

$$
\begin{aligned}
& \boldsymbol{H}_{i}=\left[\begin{array}{ccc}
\mathbf{s}_{i} & 0 & 0 \\
0 & \mathbf{s}_{i} & 0 \\
0 & 0 & \mathbf{s}_{i}
\end{array}\right], \boldsymbol{\theta}^{T}=\left[\begin{array}{lll}
A_{R} & A_{G} & A_{B}
\end{array}\right], \\
& \boldsymbol{C}_{\boldsymbol{R} G B}=\Lambda \otimes \mathbf{C}
\end{aligned}
$$

C represents the covariance matrix that takes into account spatial correlation, and $\Lambda$ is the correlation matrix between the three-color components. The symbol $\otimes$ represents kronecker product between matrices. Note that spatial correlation is assumed equal in the three components.

The decision function has to be minimized over the possible symbols $\boldsymbol{s}_{i}$, and $\boldsymbol{\theta}$. It is easy to show that this is completely equivalent to maximize the decision function over $i$ :

$$
\begin{aligned}
\Psi_{i} & =r^{T} C_{R G B}^{-1} H_{i} \hat{\theta}_{i} \\
& =r^{T} C_{R G B}^{-1} H_{i}\left(H_{i}^{T} C_{R G B}^{-1} H_{i}\right)^{-1} H_{i}^{T} C_{R G B}^{-1} r
\end{aligned}
$$

A first-order Markov model is assumed for $\boldsymbol{C}$ and although a closed form of the inverse can be derived, it is more convenient to use a pre-whitening filter, playing this role $C^{1 / 2}$. Analogously $\Lambda^{-1 / 2}$ represents the pre-whitening among the RGB components. Thus, the decision function is simplified to:

$$
\begin{aligned}
& \Psi_{i}=\frac{\left|\mathbf{r}_{w R}{ }^{T} \mathbf{s}_{s w i}\right|^{2}+\left|\mathbf{r}_{w G}{ }^{T} \mathbf{s}_{s w i}\right|^{2}+\left|\mathbf{r}_{w B}{ }^{T} \mathbf{s}_{s w i}\right|^{2}}{\mathbf{s}_{s w i}^{T} \mathbf{s}_{s w i}}, \\
& \mathbf{r}_{w R, G, B}=(\boldsymbol{\Lambda} \otimes \mathbf{C})^{-1 / 2} \mathbf{r}_{R, G, B}, \\
& \mathbf{s}_{s w i}=\mathbf{C}^{-1 / 2} \mathbf{s}_{i}
\end{aligned}
$$

$\boldsymbol{r}_{w X}$ implies a whitening of the $\boldsymbol{X}$ component of the watermarked image in the spatial and color dimension and $\boldsymbol{s}_{\boldsymbol{s} w i}$ is the symbol $\boldsymbol{s}_{i}$ that is whitened in the spatial dimension only.

\subsection{Cauchy detector}

Although a Gaussian model yields to reasonable solutions, the distribution of the whitened image tends to exhibit a slower decay in the tails of the distribution. We can model this behavior with alpha-stable distributions [6], which concentrate around the mean but are also characterized by heavier tails. In particular we consider a Cauchy distribution. Figure 1 shows the distribution of the whitened RGB components for a certain image. As it can be seen the Cauchy distribution is closer to the histogram than the Gaussian distribution. 


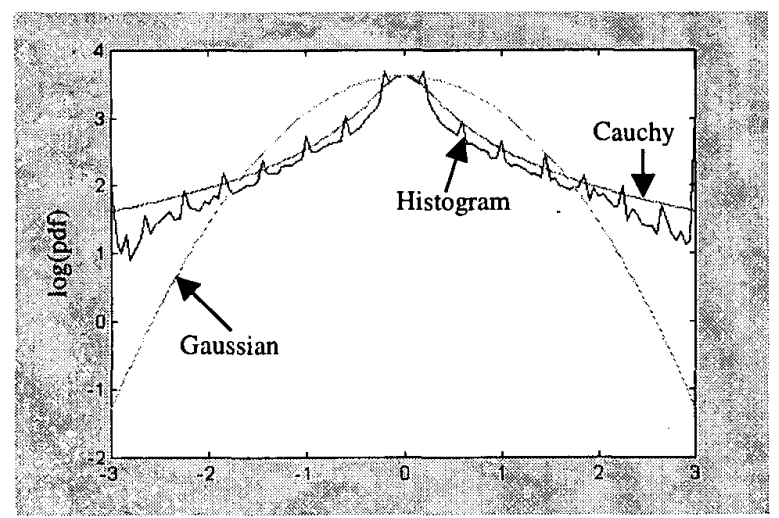

Figure 1: Histogram of the whitened R component, Gaussian pdf and Cauchy pdf for a given image

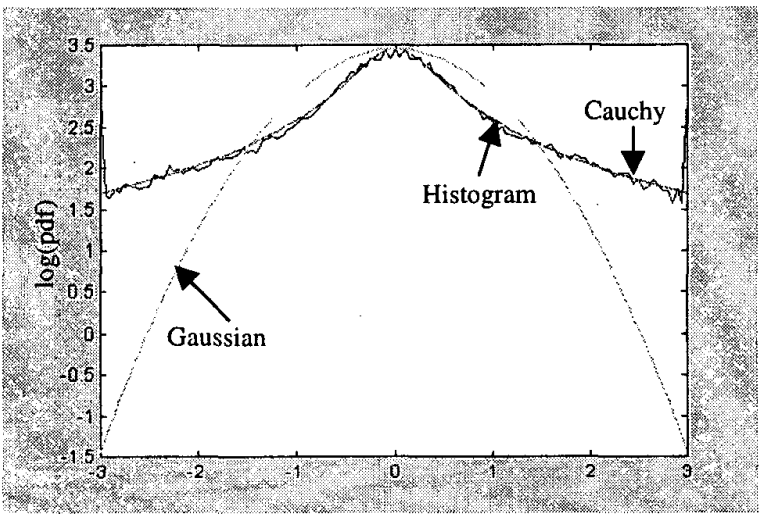

Figure 2: Histogram of the whitened G component, Gaussian pdf and Cauchy pdf for a given image

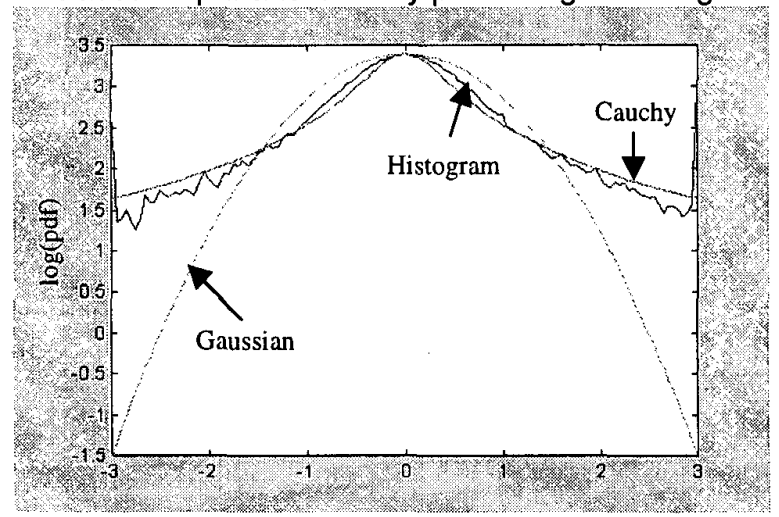

Figure 3: Histogram of the whitened B component, Gaussian pdf and Cauchy pdf for a given image
Considering the Cauchy distribution, we derive an optimum receiver in the maximum likelihood sense to decide which symbol is present in a region of the watermarked image, that is:

$$
\Psi_{i}^{C}=\min _{s_{i}} \sum_{k=1}^{N} \log \left[\gamma^{2}+z_{i}(k)^{2}\right]
$$

where $\mathrm{N}$ is the number of pixels in the block in which a symbol is present, $g$ is the distribution dispersion (it can be estimated according to the lines in [8] on the whitened components) and $z_{i}$ stands for:

$z_{i}=C_{R G B}^{-1 / 2}\left(r-H_{i}^{D} \boldsymbol{\theta}\right)=r_{w}-\frac{1}{\mathbf{s}_{w s i}^{T} \mathbf{s}_{w s i}}\left[\begin{array}{l}\mathbf{s}_{w s i} \mathbf{s}_{w s i}^{T} \mathbf{r}_{w R} \\ \mathbf{s}_{w s i} \mathbf{s}_{w s i}^{T} \mathbf{r}_{w G} \\ \mathbf{s}_{w s i} \mathbf{s}_{w s i}^{T} \mathbf{r}_{w B}\end{array}\right]$

This detector will greatly improve the probability of error with respect to the Gaussian.

\section{Results}

In order to assess the performance of the different detectors, the error rate has been computed by marking several color images with a set of symbols. First, RGB components are watermarked using the perceptive model based on Gabor filters. Table 1 shows the rate between misreceived characters and total number of detections. The same procedure is applied to the luminance component only in order to compare both watermarking strategies. Table 3 and 4 show results when applying JPEG compression. As it can be seen, in general, it is better to watermark the RGB components than the luminance component, specially under JPEG compression. On the other side, the Cauchy detector gives better results compared to the optimum linear detector. In all cases the original image and the marked image were visually equivalent.

Table 1: Error rate watermarking the RGB components $(\mu=0.8)$

\begin{tabular}{|c|c|c|c|}
\hline & Bridge & Mandril & Sfbay \\
\hline Optimum Linear, $\Psi$ & $5 / 248$ & $7 / 248$ & $0 / 248$ \\
\hline Cauchy, $\Psi^{\mathrm{c}}$ & $0 / 248$ & $4 / 248$ & $0 / 248$ \\
\hline
\end{tabular}


Table 2: Error rate watermarking the luminance component $(\mu=0.8)$

\begin{tabular}{|c|r|r|r|}
\hline & \multicolumn{1}{|c|}{ Bridge } & \multicolumn{1}{c|}{ Mandril } & \multicolumn{1}{c|}{ Sfbay } \\
\hline Optimum Linear & $5 / 248$ & $0 / 248$ & $0 / 248$ \\
\hline Cauchy & $5 / 248$ & $0 / 248$ & $0 / 248$ \\
\hline
\end{tabular}

Table 3: Error rate watermarking the RGB components under JPEG compression $(\mu=0.8)$

\begin{tabular}{|c|c|c|c|c|c|c|}
\hline JPEG & \multicolumn{2}{|c|}{ bridge } & \multicolumn{2}{c|}{ mandril } & \multicolumn{2}{c|}{ sfbay } \\
\hline & $\Psi^{\mathrm{c}}$ & $\Psi$ & $\Psi^{\mathrm{c}}$ & $\Psi$ & $\Psi^{\mathrm{c}}$ & $\Psi$ \\
\hline $\mathrm{Q}=50$ & $26 / 248$ & $35 / 248$ & $4 / 248$ & $18 / 248$ & $0 / 248$ & $0 / 248$ \\
\hline $\mathrm{Q}=70$ & $3 / 248$ & $11 / 248$ & $11 / 248$ & $14 / 248$ & $0 / 248$ & $0 / 248$ \\
\hline $\mathrm{Q}=90$ & $0 / 248$ & $14 / 248$ & $48 / 248$ & $81 / 248$ & $1 / 248$ & $5 / 248$ \\
\hline
\end{tabular}

Table 4: Error rate watermarking the luminance component under JPEG compression $(\mu=0.8)$

\begin{tabular}{|c|c|c|c|c|c|c|}
\hline JPEG & \multicolumn{2}{|c|}{ bridge } & \multicolumn{2}{c|}{ mandril } & \multicolumn{2}{c|}{ sfbay } \\
\hline & $\Psi^{\mathrm{c}}$ & $\Psi$ & $\Psi^{\mathrm{c}}$ & $\Psi$ & $\Psi^{\mathrm{c}}$ & $\Psi$ \\
\hline $\mathrm{Q}=50$ & $34 / 248$ & $35 / 248$ & $55 / 248$ & $64 / 248$ & $17 / 248$ & $24 / 248$ \\
\hline $\mathrm{Q}=70$ & $21 / 248$ & $22 / 248$ & $61 / 248$ & $59 / 248$ & $7 / 248$ & $15 / 248$ \\
\hline $\mathrm{Q}=90$ & $3 / 248$ & $7 / 248$ & $51 / 248$ & $30 / 248$ & $2 / 248$ & $2 / 248$ \\
\hline
\end{tabular}

The watermark generation scheme based on SCIELAB is shown in figure 4 .

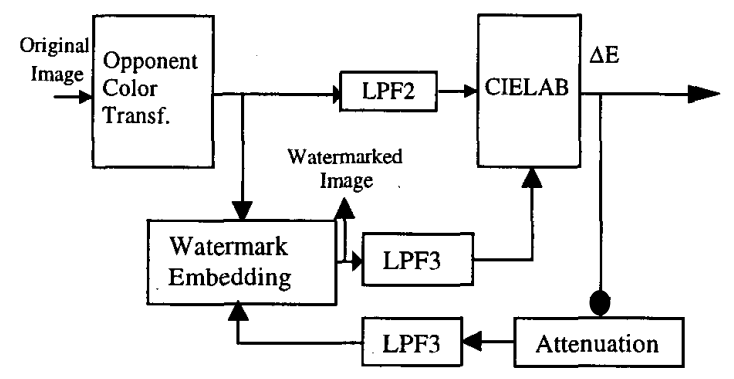

Figure 4: Watermark Embedding using S-CIELAB

The watermark is applied to the opponent color components, which are yellow-blue (YB), red-green (RG) and luminance $(\mathrm{L})$. Nevertheless numerical differences that correspond to perceptual differences are measured in the Lab space. Thus, for each pixel the perceptual difference between the original image and the watermarked image is given by :

$$
\Delta E=\sqrt{\Delta L^{2}+\Delta a^{2}+\Delta b^{2}}
$$

Usually $\Delta E$ should be lower than 3 to be unnoticeable, a more restrictive measure of $\Delta E<1$ is sometimes used. Those pixels that are above the selected threshold are attenuated until a high percentage of pixels, usually $99 \%$, are under the threshold.

The S_CIELAB has into account the spatial structure of the image and was introduced by [4]. This explains the use of low pass filters in the watermark embedding system of figure 4. Table 5 and 6 show the rate between misreceived characters and total number of detections. $\Delta E$ was set to 1 , since a threshold of 3 showed some perceptible differences.

Table 5: Error rate watermarking the opponent color components $(\Delta=1)$

\begin{tabular}{|c|c|c|c|}
\hline & bridge & mandril & sfbay \\
\hline Optimum Linear & $0 / 248$ & $8 / 248$ & $0 / 248$ \\
\hline Cauchy & $0 / 248$ & $1 / 248$ & $0 / 248$ \\
\hline
\end{tabular}

Table 6: Error rate watermarking the opponent color components under JPEG compression $(\Delta=1)$

\begin{tabular}{|c|c|c|c|c|c|c|}
\hline JPEG & \multicolumn{2}{|c|}{ bridge } & \multicolumn{2}{c|}{ Mandril } & \multicolumn{2}{c|}{ Sfbay- } \\
\hline & $\Psi^{\mathrm{c}}$ & $\Psi$ & $\Psi^{\mathrm{c}}$ & $\Psi$ & $\Psi^{\mathrm{c}}$ & $\Psi$ \\
\hline $\mathrm{Q}=50$ & $0 / 248$ & $3 / 248$ & $80 / 248$ & $99 / 248$ & $0 / 248$ & $1 / 248$ \\
\hline $\mathrm{Q}=70$ & $0 / 248$ & $0 / 248$ & $89 / 248$ & $89 / 248$ & $0 / 248$ & $3 / 248$ \\
\hline $\mathrm{Q}=90$ & $0 / 248$ & $0 / 248$ & $101 / 248$ & $94 / 248$ & $4 / 248$ & $29 / 248$ \\
\hline
\end{tabular}

\section{Conclusions}

A watermark system for color images that relies on perceptual models has been proposed. The scheme can be applied to RGB components and opponent color components to extract perceptive information. Consequently, comparison between the original and the watermarked image shows imperceptible differences by the human eye. An optimum detector has been developped showing lower probability of error with respect to: 1) optimum linear detector thanks to a better adaptation to the image model, and 2) luminance watermarking, due to the diversity allowed by the use of 
three color components. These observations are less evident when the image suffers high degradation via JPEG compression. Preliminary results give better performance when the S-CIELAB based model is used. Future works devises the use of more complex modulations allowing the introduction of more information in the watermark and robustness to image cropping.

\section{References}

[1]Josep Vidal, Elisa Sayrol, "Optimum Watermark Detection and Embedding in Digital Images", Proc. of IEEE Workshop on Multimedia Signal Processing, Los Angeles, CA, December 1998, pp. 285-290.

[2] J.F. Delaigle, et al., "Low Cost Watermarking Based on a Human Visual Model”, Proc. ECMAST 97, 1997.
[3] M. Kutter, F. Jordan, F. Bossen, "Digital watermarking of color images using amplitude modulation, Journal of Electronic Imaging 7(2), pp. 326332, April 1998.

[4]David J. Fleet, David J. Heeger, "Embedding Invisible Information in Color Images", Proc. of ICIP '97, Santa Barbara, October 1997, pp 532-535

[5] I. J. Cox, et al., "Secure Spread Spectrum Watermarking for Multimedia", IEEE Trans. on Image Processing, vol. 6, no 12, Dec. 1997

[6]M. Shao and C. L. Nikias, "Signal Processing with fractional Lower Order Moments: Stable Processes and their Applications", Proc. of IEEE, V-81, No. 7, July 93.

[7]G. A. Tsihrintzis and C. L. Nikias, "Performance of Optimum and Suboptimum Receivers in the Prsence of Impulsive Noise Modeled as an Alpha-Stable Process", IEEE Trans. on Comm., V-43, No. 2/3/4 February 1995. 